Research Paper

\title{
High-expression of DJ-I and Loss of PTEN Associated with Tumor Metastasis and Correlated with Poor Prognosis of Gastric Carcinoma
}

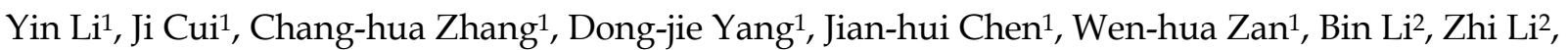
Yu-long $\mathrm{He}^{1 凶}$

1. Department of Gastrointestinal Surgery, The First Affiliated Hospital, Sun Yat-sen University, 58, Zhongshan Road II, Guangzhou 510080, China;

2. Department of Pathology, The First Affiliated Hospital, Sun Yat-sen University, 58, Zhongshan Road II, Guangzhou 510080, China.

$\square$ Corresponding author: Professor Yu-long He, Ph.D, M.D. Department of Gastrointestinal Surgery, The First Affiliated Hospital, Sun Yat-sen University, 58, Zhongshan Road II, Guangzhou 510080, China. Tel: (8620) 87755766-8211 Email: ylh@medmail.com.cn.

(c) Ivyspring International Publisher. This is an open-access article distributed under the terms of the Creative Commons License (http://creativecommons.org/ licenses/by-nc-nd/3.0/). Reproduction is permitted for personal, noncommercial use, provided that the article is in whole, unmodified, and properly cited.

Received: 2013.07.30; Accepted: 2013.09.15; Published: 2013.09.24

\begin{abstract}
Background and aims: DJ-I and PTEN have been shown to involve in multiple cell processes and play an important role in cancer development and progression. However, their relationship with gastric carcinoma (GC) has not been identified yet. The purpose of this study is to clarify the relationship of DJ-I and phosphatase and tensin homolog (PTEN) with clinicopathological parameters and prognosis in GC.

Methods: II4 specimens were collected from GC patients and expression of DJ-I and PTEN in tissue microarray was evaluated by immunohistochemical staining. Correlation between immunostainings and clinicopathological parameters, follow-up data of patients, was analyzed statistically.

Results: High expression of $D J-I$ was found in $66.7 \%(76 / / 14)$ and associated with tumor depth $(P=0.003)$, lymph node metastasis $(P=0.0 \mathrm{II})$, distant metastasis $(P=0.00 \mathrm{I})$ and advanced clinical stage $(P=0.00 I)$. Loss or downregulation of PTEN was found in $58.7 \%(67 / / 14)$ and associated with advanced clinical stage $(P=0.018)$ and high expression of $D J-I$ in tumor cells $(P=0.006)$. In univariate survival analysis, high-expression of DJ-I or loss of PTEN was significantly associated with poor prognosis of GC patients. However, only tumor depth $(P=0.0 I I)$ and coexistence of $D J-I$ and PTEN abnormal expression $(P=0.009)$ emerged as strong independent prognostic factors for overall survival of GC patients.

Conclusions: the present study indicates that $\mathrm{DJ}-\mathrm{I}$ and PTEN may play their roles in progression of GC in a cooperating pattern. Co-existence of abnormal DJ-I and PTEN expression is likely to serve as an independent predictive factor for prognosis of GC patients.
\end{abstract}

Key words: Gastric carcinoma; DJ-1; PTEN; Metastasis; Prognosis.

\section{Introduction}

Gastric carcinoma (GC) is one of the most common causes of cancer-related deaths in the world, and over one-third of all GCs worldwide occur in China [1-2]. Although the combination of surgical resection and adjuvant chemo- or radiotherapy has been applied widely in China, the 5 -year survival rate of patients with GC is currently less than $20 \%$ because of the frequency of distant metastases and local recurrence. When metastasis has occurred, the patients are often unsuitable for curative surgery. Metastasis is the main cause of treatment failure and induces a poor prognosis in patients with GC [3-4]. In the past two decades, various researches on GC have been preformed and tried to find the mechanism of 
invasion and metastasis of this tumor, but the precise molecular mechanism remains to be clarified. In fact, whether or not certain molecules involved in the invasion and metastasis of GC, and consequently influenced the prognosis of GC is largely unknown.

Phosphatase and tensin homolog deleted on chromosome 10 (PTEN) is a tumor suppressor, also known as a key negative regulator of the phosphatidylinositol 3-kinase (PI3K)-protein kinase B (PKB/Akt) signaling pathway [5]. It has been demonstrated that PTEN affects processes such as cell cycle progression, apoptosis, migration, metabolism, transcription and translation by negatively regulating the AKT pathway and decreasing phosphorylation of AKT substrates [6]. PTEN can also inhibits cell invasion and metastasis [7-8], as well as restricting cell differentiation [9]. The loss or downregulation of PTEN appears to be a common event in many types of tumors. Loss of PTEN have been associated with invasive urothelial carcinoma of urinary bladder [10]. The PTEN gene was previously reported to be transcriptionally silenced by promoter methylation in a number of GC cases [11], and loss or reduced expression of PTEN correlated with advanced-stage GCs [12]. However, the role of the loss or reduced expression of PTEN in GC's prognosis remains unclear.

DJ-1, also known as the Parkinson's disease-associated protein 7 (PARK7), is a 189 amino acid protein with multiple functions. Accumulating evidence has shown that DJ-1 is overexpressed in many types of malignant tumors [13-14] and also correlated with tumor progression in various cancers [15-16]. DJ-1 promotes cell survival by modulating PTEN [17], and high DJ-1 levels have been reported during initiation and progression in certain types of cancer [18-19]. Elevated serum concentrations of DJ-1 in GC patients in high-incidence regions of gastric cancer has suggested that differing mechanisms of disease pathogenesis may be at play in high- and low-incidence regions of this tumor [20]. However, the role of DJ-1 in cancer metastasis, especially its correlation to the prognosis of GC remains unclear.

In the present study, we evaluate the expression of DJ-1 and PTEN in GCs and investigate their association with clinicopathological parameters to determine the role of these proteins in the prognostic significance of GC.

\section{Material and Methods}

\section{Specimens of GC and Clinicopathological Findings}

Archival formalin-fixed, paraffin-embedded specimens from 114 primary GC patients during
2004-2007 in the first Affiliated Hospital of Sun Yat-sen University (Guangzhou, China) were collected. The patients were 72 males and 42 females with a median age of 55 years (range 25-80). According to $\mathrm{WHO}$ histological classification, all patients were diagnosed as gastric adenocarcinoma with varied tumor differentiation [21]. The patients were classified the disease stage according to the criteria of the American Joint Committee on Cancer (AJCC) and the International Union for Cancer Control (UICC) 2010 cancer staging manual [22], and 40 in early clinical stage (Stage I and II), 74 in advanced clinical stage (stage III and IV). All the patients were treated with radical surgical resection (D2). All patients except Stage I patients were also treated with standard adjuvant chemotherapy of modified FOLFOX6 regimen (oxaliplatin, $85 \mathrm{mg} / \mathrm{m}^{2}$ in $250 \mathrm{ml}$ of dextrose $5 \%$, concurrent with LV, $400 \mathrm{mg} / \mathrm{m}^{2}$, given as a 2-hr intravenous infusion on day 1 , followed by a bolus $5-\mathrm{FU}, 400 \mathrm{mg} / \mathrm{m}^{2}$, on day 1 and a continuous $5-\mathrm{FU}$, $1,200 \mathrm{mg} / \mathrm{m}^{2} /$ day, on day 1 and $2,46-\mathrm{hr}$ continuous infusion of a total $5-\mathrm{FU}$ of $2,400 \mathrm{mg} / \mathrm{m}^{2}$ )

\section{Paraffin Tissue Microarray Construction}

In this study, the paraffin tissue microarray construction was conducted by pervious description [23]. Briefly, section was cut from each paraffin block and stained with hematoxylin and eosin (H\&E). Each donor block was overlaid with the corresponding H\&E slide and observed by experienced pathologists. The area in donor block for tissue microarray sampling was verified according to the H\&E slide and marked. A manual tissue arrayer (Beecher Instruments Inc., Sun Prairie, WI, USA) was used for array construction. Two representative $1.0 \mathrm{~mm}$ cores were removed from each donor block and transferred to pre-molded recipient paraffin block with designed orientation. An additional six cores derived from tonsil, lymph node and breast carcinoma were used as control material. The serial sections with $4-\mu \mathrm{m}$ thickness were cut from arrayed block and mounted on APES-coated glass slides and stored at $4^{\circ} \mathrm{Cfor}$ further detection.

\section{Immunohistochemistry and Scoring}

The tissue array sections were subjected to immunostaining using a ChemMate Envision/HRP Kit (Dako Cytomation, Glostrup, Denmark). Slides were deparaffinized in xylene and rehydrated in decreasing concentrations of ethanol and rinsed in phosphate buffered saline. The slides were incubated with hydrogen peroxide for $10 \mathrm{~min}$ following microwave with $10 \mathrm{mM}$ citrate buffer $(\mathrm{pH}$ 6.0; Sigma-Aldrich, Munich, Germany) at 5-min intervals for a total of $10 \mathrm{~min}$. After blocking with normal se- 
rum for $10 \mathrm{~min}$, the slides were incubated with a 1:100 dilution of the rabbit anti-human polyclonal DJ-1 antibody (FL-189, Santa Cruz biotechnology, Dallas, TX, USA), or a 1:100 dilution of rabbit anti-human polyclonal PTEN antibody (Cell Signaling technology, Beverly, MA, USA) for $60 \mathrm{~min}$, respectively. Slides were detected by ChemMate Envision/HRP Kit for 30 min at room temperature and followed by developing with diaminobenzidine (DAB) for visualization. Negative controls were carried out by substituting non-immune serum for the primary antibodies. The immunostaining results were evaluated and scored semiquantitatively by two pathologists without knowledge of the clinical data of patients. Evaluation of the immunostaining results was performed by stain intensity and stain area (double scoring system) as previously described [16]: score 0 , no staining; score 1 , weak staining; score 2, moderate staining; score 3 , strong staining. Staining area less than $35 \%$ (score 1); $35 \%-75 \%$ (score 2 ); more than $75 \%$ (score 3 ) of tumor cells. The high-expression of DJ-1 and PTEN was defined when immunostaining score was equal or more than 4, whereas low-expression of proteins was less than score 4. Dual abnormal expression of DJ-1 and PTEN was defined when the tumor showed high expression of DJ-1 and low or loss expression of PTEN simultaneously.

\section{Statistical Analysis}

All statistical analysis was carried out by SPSS 13.0 software for Windows. Chi-square test was used to assess DJ-1 and PTEN expression with clinicopathological characteristics. The survival curve of patients was determined by Kaplan-Meier method and Cox regression, and statistical evaluation was performed using the log rank test. A P-value $<0.05$ was considered statistically significant.

\section{Results}

\section{Clinical findings of the patients with GC}

The clinicopathological characteristics of the 114 GC patients are recorded in Table 1. All of patients were treated in our hospital with complete follow-up data. There were 72 males and 42 females with a median age of 55 years (range from 25 to 80 years old). Histological grade was used to assess differentiation stage according to the WHO criteria for tumor classification of gastrointestinal tumor. 67 patients had carcinomas with well or moderate differentiation, and the other 47 patients had poorly differentiated carcinoma. AJCC/UICC tumor stage was used to stage the primary GCs. The patients with stage I or II were categorized as early clinical stage, while stage III or IV were advanced clinical stage. Depth of invasion was assessed using AJCC/UICC criteria, and 13, 13, 75 and 13 patients were classified as stage T1, T2, T3 and $\mathrm{T} 4$, respectively. T3 or T4 was identified as deeper tumors. Lymph node metastasis and distant metastasis were assessed using AJCC/UICC criteria. The mean duration of follow-up was 32.4 months (range from 2 to 64 months).

\section{DJ-I and PTEN expression in correlation to clinicopathological parameters in GC}

DJ-1 expression in GC was mostly cytoplasmic with some nuclear and membranous staining (Fig.1A and Fig.1B). High DJ-1 was expressed in 66.7\% (76/114) of GCs. The immunostaining pattern of PTEN was also characterized by cytoplasmic and nuclear staining of the carcinoma (Fig.1C). High PTEN expression was detected in $41.2 \%(47 / 114)$ of carcinomas, while downregulation or loss of PTEN expression was found in most of GCs $(58.7 \%, 67 / 114)$ (Fig.1D). Table 1 shows the correlation between markers analyzed and clinically important histopathological features of the tumors. The higher expression of DJ-1 was significantly correlated with the deeper tumor $(\mathrm{P}=0.003)$, lymph node metastasis $(\mathrm{p}=0.011)$, distant metastasis $(\mathrm{P}=0.001)$ and advanced clinical stage $(\mathrm{P}=0.001)$. However, loss expression of PTEN was only found to correlated with distant metastasis $(\mathrm{P}=0.001)$ and advanced clinical stage $(\mathrm{P}=0.018)$. Furthermore, High DJ-1 expression in tumor cells correlated negatively with downregulation or loss expression of PTEN in tumor $(\mathrm{P}=0.001)$.

\section{Correlation of proteins expression and clini- copathological features with survival of GC patients}

In this study, 62 (35.7\%) patients with GC died of tumor in 5-year survival follow-up. In univariate analysis, the survival rates of patients were associated with tumor depth, lymph node metastasis, distant metastasis, advanced clinical stage, high-expression of DJ-1 and loss or downregulation of PTEN (Table 2 and Fig.2). However, in multivariate analysis, only tumor depth (hazard ratio, 15.198; 95\% confidence interval, 1.889-122.233, $\mathrm{P}=0.010$ ) and abnormal expression of DJ-1 and PTEN of GC tumor cells (hazard ratio, 8.074; 95\% confidence interval, 1.655-36.369, $\mathrm{P}=0.009$ ) were independent risk factors for poor overall survival. Interestingly, histologically poorly differentiated carcinoma was also observed to be an independent risk factor for GC patients. However, DJ-1 or PTEN expression status alone was not associated with overall survival of patients with GC (Table 3). 
Table I. Correlation between proteins expression and clinicopathological parameters of GC patients (Chi-square test).

\begin{tabular}{|c|c|c|c|c|c|c|}
\hline \multirow[t]{2}{*}{ Variable } & \multicolumn{2}{|c|}{ DJ-1 expression } & \multicolumn{2}{|c|}{ PTEN expression } & \multicolumn{2}{|c|}{ Abnormal expression of DJ-1 and PTEN } \\
\hline & Low $(n=38)$ & High $(n=76)$ & Low $(n=67)$ & High $(n=47)$ & No $(n=63)$ & Yes $(n=51)$ \\
\hline \multicolumn{7}{|l|}{ Age (year) } \\
\hline$<55(n=53)$ & 17 & 36 & 32 & 21 & 26 & 27 \\
\hline \multirow{2}{*}{$\geq 55(\mathrm{n}=61)$} & 21 & 40 & 35 & 26 & 37 & 24 \\
\hline & $\mathrm{P}=0.806$ & & $\mathrm{P}=0.782$ & & $P=0.206$ & \\
\hline \multicolumn{7}{|l|}{ Gender } \\
\hline Male $(n=72)$ & 24 & 48 & 41 & 31 & 43 & 29 \\
\hline \multirow[t]{2}{*}{ Female $(n=42)$} & 14 & 28 & 26 & 16 & 20 & 22 \\
\hline & $\mathrm{P}=1.000$ & & $\mathrm{P}=0.647$ & & $\mathrm{P}=0.211$ & \\
\hline \multicolumn{7}{|l|}{ Tumor differentiation } \\
\hline Well and moderate $(n=67)$ & 21 & 46 & 37 & 30 & 36 & 31 \\
\hline \multirow[t]{2}{*}{ Poor $(n=47)$} & 17 & 30 & 30 & 17 & 27 & 20 \\
\hline & $\mathrm{P}=0.541$ & & $\mathrm{P}=0.409$ & & $\mathrm{P}=0.750$ & \\
\hline \multicolumn{7}{|l|}{ Tumor depth } \\
\hline $\mathrm{T} 1-2(\mathrm{n}=26)$ & 14 & 12 & 12 & 14 & 20 & 6 \\
\hline \multirow[t]{2}{*}{$\mathrm{T} 3-4(\mathrm{n}=88)$} & 24 & 64 & 55 & 33 & 43 & 45 \\
\hline & $\mathrm{P}=0.003$ & & $\mathrm{P}=0.103$ & & $\mathrm{P}=0.001$ & \\
\hline \multicolumn{7}{|l|}{ Lymph node metastasis } \\
\hline N0 $(n=40)$ & 19 & 21 & 20 & 20 & 28 & 12 \\
\hline \multirow[t]{2}{*}{$\mathrm{N} 1-3(\mathrm{n}=74)$} & 19 & 55 & 47 & 27 & 35 & 39 \\
\hline & $\mathrm{P}=0.011$ & & $\mathrm{P}=0.190$ & & $\mathrm{P}=0.012$ & \\
\hline \multicolumn{7}{|l|}{ Distant metastasis } \\
\hline M0 (n=92) & 36 & 56 & 47 & 45 & 61 & 31 \\
\hline \multirow[t]{2}{*}{ M1 $(n=22)$} & 2 & 20 & 20 & 2 & 2 & 20 \\
\hline & $\mathrm{P}=0.001$ & & $\mathrm{P}=0.001$ & & $\mathrm{P}=0.001$ & \\
\hline \multicolumn{7}{|l|}{ Clinical stage } \\
\hline Early stage $(n=40)$ & 21 & 19 & 17 & 23 & 32 & 8 \\
\hline \multirow[t]{2}{*}{ Advanced stage $(\mathrm{n}=74)$} & 17 & 57 & 50 & 24 & 31 & 43 \\
\hline & $\mathrm{P}=0.001$ & & $\mathrm{P}=0.018$ & & $\mathrm{P}=0.001$ & \\
\hline \multicolumn{7}{|l|}{ PTEN expression } \\
\hline Low expression $(\mathrm{n}=67)$ & 16 & 51 & & & & \\
\hline \multirow[t]{2}{*}{ High expression $(\mathrm{n}=47)$} & 22 & 25 & & & & \\
\hline & $\mathrm{P}=0.006$ & & & & & \\
\hline
\end{tabular}

Table 2. Kaplan-Meier analysis for overall survival rate of patients with GC.

\begin{tabular}{|c|c|c|c|}
\hline Characteristics & Mean survival time (months) & 95\% confidence interval (months) & $P$ values* \\
\hline Age (years) & & & 0.843 \\
\hline$<55$ & $35.7 \pm 3.24$ & 29.4-42.1 & \\
\hline$\geq 55$ & $39.7 \pm 3.08$ & $33.7-45.8$ & \\
\hline Gender & & & 0.110 \\
\hline Male & $41.3 \pm 2.98$ & $35.5-47.1$ & \\
\hline Femal & $33.9 \pm 3.64$ & $26.7-41.0$ & \\
\hline Tumor differentiation & & & 0.224 \\
\hline Well and moderate & $38.8 \pm 2.91$ & $33.1-44.5$ & \\
\hline Poor & $36.5 \pm 3.55$ & $29.5-43.5$ & \\
\hline Tumor depth & & & 0.001 \\
\hline $\mathrm{T}_{1-2}$ & $61.7 \pm 3.12$ & $55.6-67.8$ & \\
\hline $\mathrm{T}_{3-4}$ & $32.3 \pm 2.43$ & $27.5-37.1$ & \\
\hline Lymph node metastasis & & & 0.004 \\
\hline $\mathrm{N}_{0}$ & $50.6 \pm 3.61$ & $43.6-57.7$ & \\
\hline $\mathrm{N}_{1-3}$ & $32.2 \pm 2.65$ & 26.9-37.4 & \\
\hline \multicolumn{4}{|l|}{ Distant metastasis } \\
\hline $\mathrm{M}_{0}$ & $43.4 \pm 2.54$ & $38.4-48.4$ & 0.001 \\
\hline $\mathrm{M}_{1}$ & $18.2 \pm 3.33$ & $11.6-24.7$ & \\
\hline Clinical stage & & & 0.001 \\
\hline Early stage (I/II) & $55.8 \pm 2.88$ & $50.2-61.5$ & \\
\hline Advance stage (III/IV) & $29.8 \pm 2.63$ & $24.6-35.0$ & \\
\hline DJ-1 expression & & & 0.011 \\
\hline Low expression & $46.9 \pm 4.57$ & 37.9-55.9 & \\
\hline High expression & $34.9 \pm 2.51$ & $30.1-39.9$ & \\
\hline PTEN expression & & & 0.007 \\
\hline Low expression & $33.3 \pm 3.02$ & $27.3-39.2$ & \\
\hline High expression & $46.1 \pm 3.07$ & $40.0-52.1$ & \\
\hline Abnormal expression of DJ-1 and PTEN & & & 0.001 \\
\hline No & $48.6 \pm 2.92$ & 42.9-54.4 & \\
\hline Yes & $26.7 \pm 2.91$ & 21.0-32.4 & \\
\hline
\end{tabular}


Table 3. Cox regression model for multivariate analyses of prognostic factor in GC.

\begin{tabular}{llll}
\hline Variable & Hazard ratio & $95 \%$ confidence interval & $P$ value \\
\hline Age $(<55$ vs. $\geq 55)$ & 1.132 & $0659-1.944$ & 0.652 \\
Gender (male vs. female) & 1.057 & $0.615-1.815$ & 0.840 \\
Tumor differentiation (well \& moderate vs. poor) & 1.774 & $1.038-3.032$ & 0.035 \\
Tumor depth ( $\mathrm{T}_{1-2}$ vs. $\mathrm{T}_{3-4}$ ) & 15.198 & $1.889-122.233$ & 0.011 \\
Lymph node metastasis $\left(\mathrm{N}_{0}\right.$ vs. N $\left.\mathrm{N}_{1-3}\right)$ & 1.235 & $0.411-3.710$ & 0.705 \\
Distant metastasis $\left(\mathrm{M}_{0}\right.$ vs. $\mathrm{M}_{1}$ ) & 1.524 & $0.792-2.935$ & 0.206 \\
Clinical stage (Stage I-II vs. III-IV) & 1.347 & $0.344-5.274$ & 0.668 \\
DJ-1 expression (low vs. high) & 0.353 & $0.121-1.028$ & 0.056 \\
PTEN expression (low vs. high) & 2.864 & $0.773-10.610$ & 0.115 \\
Abnormal expression of DJ-1 and PTEN (no vs.yes) & 9.073 & $1.655-39.369$ & 0.009 \\
\hline
\end{tabular}
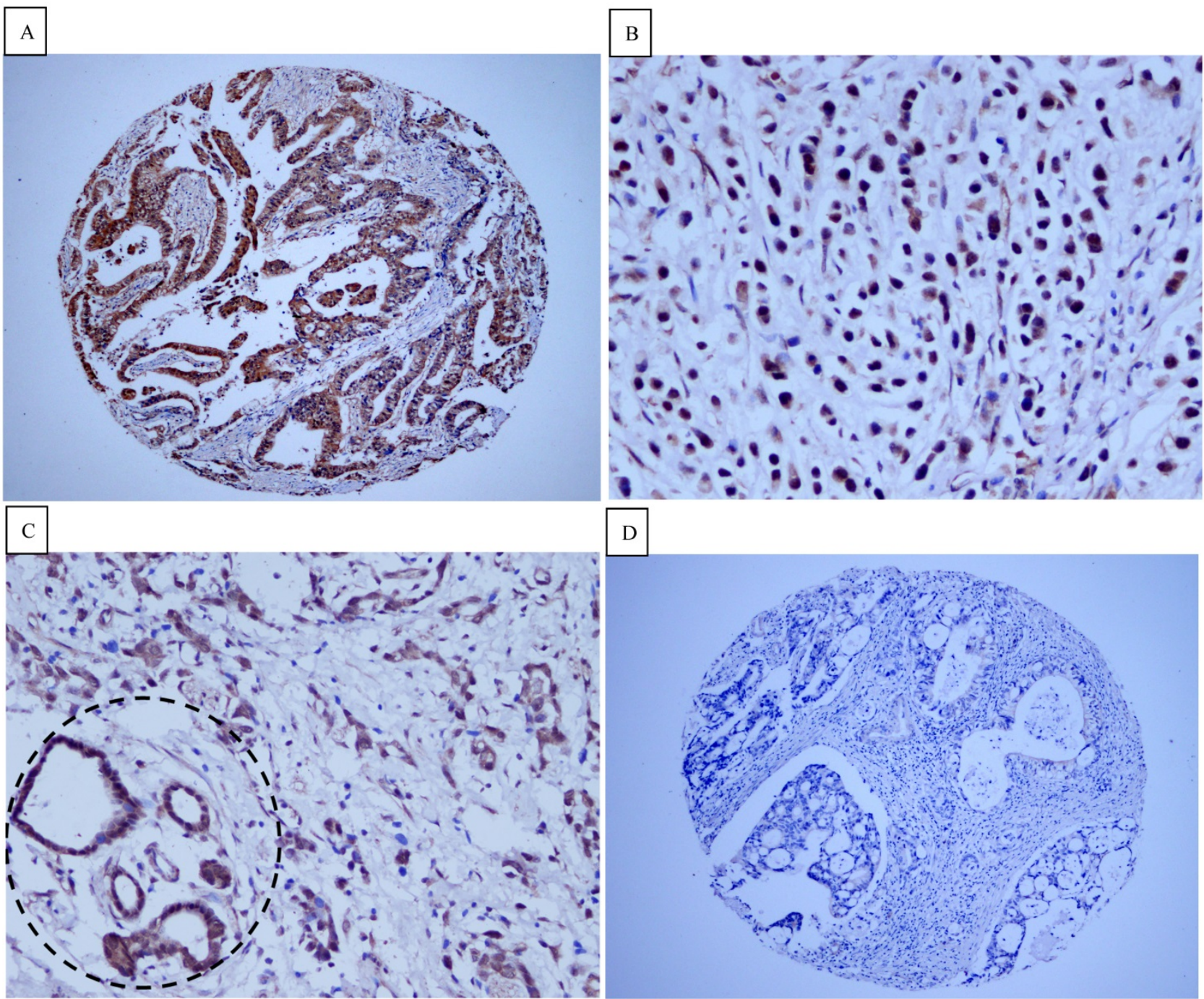

Fig I. Immunohistochemical staining of GC tissue microarray. (a) High expression of DJ-I in the cytoplasm of tumor cells was shown under lower power field. (b) In some GCs, nuclear and cytoplasmic positive staining of DJ-I in the tumor cells could be also observed. (c) High expression of PTEN could be found in tumor cells of some GCs, while, the adjacent non-neoplastic glands (circled with dotted line) showed strongly and diffusely positive staining of PTEN in cytoplasm and nuclear. (d) However, in most of GCs, PTEN expression was entirely loss or remarkably reduced. (a and d, immunohistochemical staining with original magnification $\times 100 ; b$ and $c$, immunohistochemical staining with original magnification $\times 400$ ). 

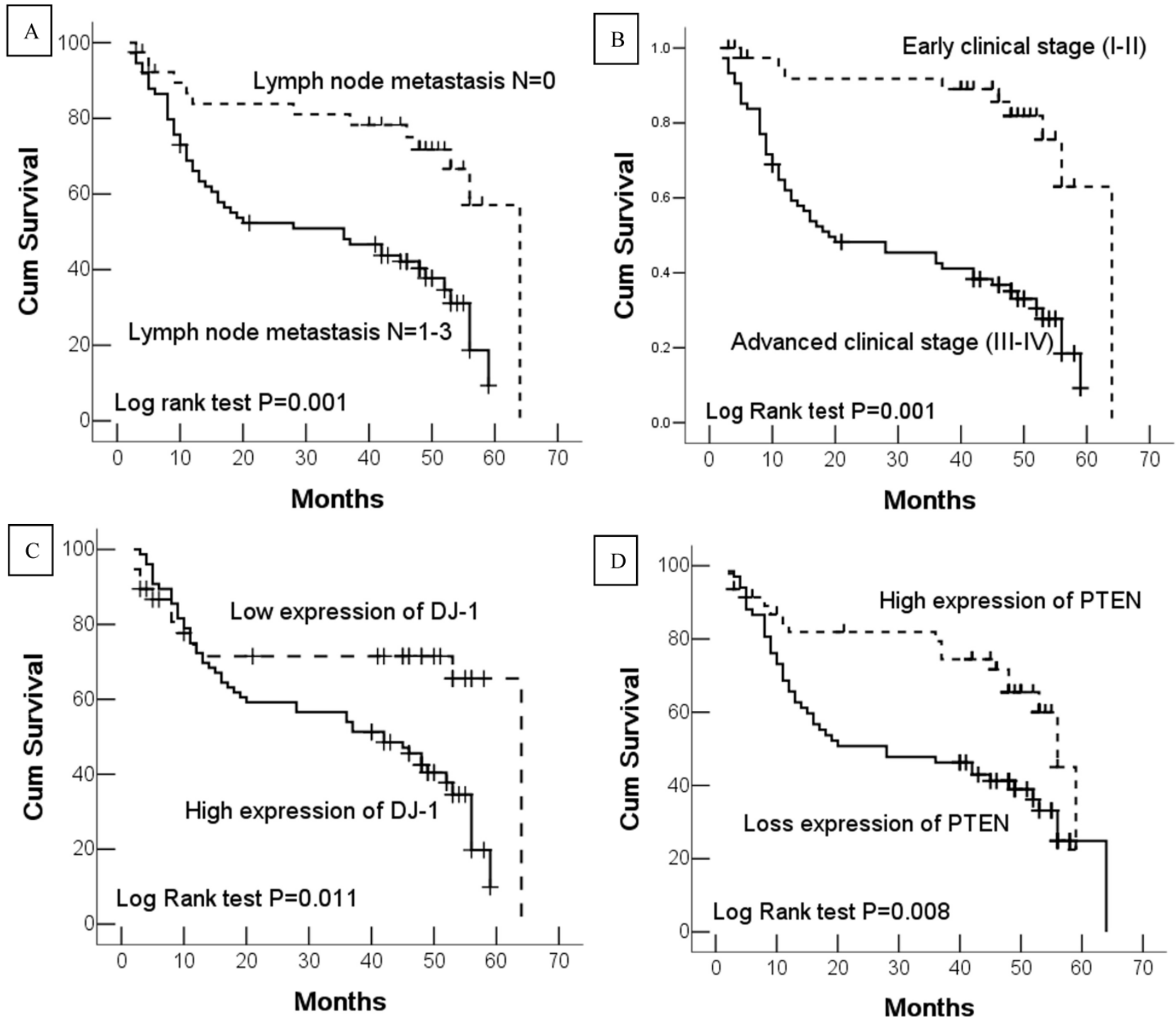

Fig 2. Kaplan-Meier survival analyses of GC patients. Kaplan-Meier curve showing the patients with lymph node metastasis (NI-N3) (a) and advanced clinical stage (stage III and IV) (b) have a significantly lower survival rate. A significant difference of survival rate was found between the GC patients with DJ-I (c) high-expression and its low-expression counterpart in tumor. (d) The patients with loss or reduced expression of PTEN have a significant lower survival rate than those with high expression of PTEN in tumor.

\section{Discussion}

DJ-1 is a conserved protein with multiple functions. DJ-1 has been reported to be overexpressed in several types of human cancer, including lung cancer [24], breast cancer [25], pancreatic cancer [26], esophageal carcinoma [27], and bladder tumors [10]. It has also demonstrated that DJ-1 improves tumor cell survival by modulating the Akt/PI3K axis [17], inhibiting apoptosis through repression of the p53-Bax-caspase pathway [28] and stabilizing the antioxidant transcriptional master regulator Nrf2 [29]. Downregulation of DJ-1 by transfection with small interfering RNA (siRNA) targets DJ-1 inhibited cell proliferation and enhances apoptosis of laryngeal squamous cell carcinoma (SCC) Hep-2 cells [16]. In the present study, a high expression of DJ-1 was detected in deeper tumor (T3-T4) of GCs, indicating that a high expression of DJ-1 is associated with tumor invasiveness. This result coincides with DJ-1 high expression in pT3-pT4 glottic SCCs [16] and T4 esophageal SCCs [27].

The ability to migrate and invade the basement membrane into surrounding tissues, blood and lymphatic vessels is one of the essential hallmarks of cancer and is a prerequisite for local tumor progression and metastatic spread [30]. Recent studies have indicated that DJ-1 may be related to cancer metastasis. DJ-1 expression was found to be upregulated in uveal melanoma cells that had a high degree of metastatic potential [31]. DJ-1 has also been found to be increased during the progression of cervical cancer from normal tissues and during the progression of patient-matched high-grade squamous intraepithelial 
lesions to invasive carcinoma tissues [14]. In the present study, we found that high expression of DJ-1was significantly correlated with lymph node metastasis, distant metastasis and shorter survival time of GC patients. Those results indicate that high expression of DJ-1 in GC tumor cells may play important roles in tumor progression and influence the prognosis of patients. Cancer invasion and metastasis require controlled degradation of the extracellular matrix (ECM). Recent studies have shown that DJ-1 promotes tumor cells invasion and migration by activating uPA system and MMP2 through the SRC/ERK pathway [32]. Although we did not detect the expression of ECM-related proteins in GC tumor cells, it is reasonable to believe that high expression of DJ-1, resulting in activation of enzymes for ECM degradation or dysfunction of cytoskeleton, plays important roles in metastasis and progression of GC. The DJ-1 expression status in tumor cells was valuable for prognostic evaluation of GC patients. Of course, further study is needed to address the underlining mechanism and how DJ-1 is able to regulate the cytoskeleton or ECM degradation in GCs.

The tumor suppressor PTEN is encoded by a gene that shows the greatest selection for loss in the human genome [33]. Studies have shown that the PTEN gene is frequently mutated or lost in many types of human primary carcinomas, and PTEN expression is often dysregulated in carcinoma, even in the absence of genetic loss or mutation [34]. Loss of PTEN has been demonstrated to associate with non-papillary, invasive and high-grade urothelial carcinoma, while strong expressions of PTEN are observed in the nucleus and cytoplasm in normal urothelium [10]. In GCs, PTEN expression was frequently lost in the cytoplasm. The loss of cytoplasmic PTEN expression was significantly correlated with histological grade, and the loss of nuclear or total PTEN expression was significantly correlated with tumor stage [12]. In the present study, the results showed that the loss of cytoplasmic PTEN is associated with distant metastasis and advanced clinical stage of GC patients. Therefore, a low PTEN expression may be a marker for progression of tumors and closely correlates with tumor invasiveness and metastasis. These findings indicate a novel molecular basis for the critical role of PTEN loss in the development and progression of GC. However, on multivariate analyses of prognostic factors in GC, loss of PTEN was failed to be detected as an independent factor to influence the prognosis of GC patients. Similarly, pervious study on PTEN expression in gastric cancer also showed PTEN expression was not associated with overall or 3-year survival of patients with GC [12]. These results indicate that loss of PTEN in tumor cells may contribute in part to cells invasiveness or distant metastasis and accelerate the progression of GC, but it might not be an independent prognostic factor to predict accurately the prognosis of GC.

In the present study, we found high expression of tumor cells was closely in correlation to the loss of PTEN expression in GC. Since PTEN has been identified to be modulated by DJ-1 [17], we considered that DJ-1/PTEN modulating loop in GC plays some important roles in facilitating dissemination of tumor cell from primary tumor to secondary tumor by its specific pathway. PTEN is a key negative regulator of the PI3K-protein kinase $\mathrm{B}$ (PKB/Akt) signaling pathway [5]. PI3K, activated by growth factors, catalyzes the phosphorylation of phosphatidylinositol $(4,5)$-biphosphate (PIP2) to phosphatidylinositol (3,4,5)-triphosphate (PIP3). PIP3 recruits 3-phosphoinositide-dependent kinase (PDK), which phosphorylates and activates Akt [35]. Akt is a signal transduction protein that plays a key role in multiple signaling pathways, including cell proliferation, apoptosis and transcription [36]. DJ-1 has been demonstrated to be a potent modulator of the PI3K/Akt pathway in both cancer cells and neurons [37-38]. Therefore, it is reasonable to believe the presence of DJ-1/PTEN modulating loop in GC tumor cells for their survival and other behavior. In our study, we found that only abnormal expression of DJ-1 and PTEN in tumor cells simultaneously was in correlation to a poor outcome and to be a potent prognostic factor to predict the prognosis of patients with GC. The high expression of DJ-1 or loss of PTEN alone was not an independent prognostic factor. These results indicate that DJ-1 and PTEN may play their roles in progression of GC in a cooperating pattern. It has been suggested that PTEN is required for this function of DJ-1 [17]. Downregulation of DJ-1 using siRNA resulted in the inhibition of endogenous Akt phosphorylation only in Pten+/- immortalized MEFs, but not in Pten-/- MEFs [17]. Thus, co-existence of high DJ-1 and reduced PTEN in GCs is likely to serve as a prognostic marker and potential therapeutic target. The further studies are required to take into account post-surgical treatments for such molecular targets to improve the survival rate of patients with GC.

In our study, we found deeper tumor of GC (T3 and T4) was closely associated with shorter survival time of patients both in univariate and multivariate analysis of prognostic factors in GC. This result coincided with previous studies by Kim et al. [39] indicating tumor depth of tumor invasion was significant prognostic factors of GC. The close relationship between DJ-1 and tumor depth in the study implies high expression of DJ-1 in tumor cells may be involved in 
the tumor invasiveness. In addition, our data showed interestingly that tumor histological differentiation was also an independent predictor for GC patients. Tumor differentiation appears to be independent of other clinicopathological features. To our knowledge, so far a correlation for DJ-1, PTEN expression and cell differentiation in GCs was not observed. We wonder if tumor differentiation is responsible for epithelial-mesenchymal transition (EMT) process, promotes tumor progression, and consequently influences the prognosis of GC patients, because most of tumor cells with poor differentiation appear to have loose and detached histological structure. Additional studies are required to better understand its contribution to GC tumorigenesis and prognosis.

In conclusion, although the precise factors responsible for metastasis and poor prognosis in GC have not been identified, our study indicates high expression of DJ-1 and the loss or downregulation of PTEN expression is involved in the progression of GC in a cooperating pattern and is a valuable predictor for prognosis evaluation of GC patients. DJ-1 and PTEN are promising new molecular targets for designing novel preventive and therapeutic strategies to control this malignant tumor

\section{Abbreviations}

GC: gastric carcinoma; PARK7: parkinson's disease-associated protein 7; PTEN: phosphatase and tensin homolog deleted on chromosome 10; PI3K: phosphatidylinositol 3-kinase; SCC: squamous cell carcinoma; ECM: extracellular matrix; ERK: extracellular signal-regulated kinase; uPA: urokinase plasminogen activator

\section{Acknowledgement}

This study is supported by Project of Guangdong Provincial Science and Technology (Grant number: 2011B08071048), Project of Health of Guangdong Province (Grant number: A2011160), and Program of National Key Clinical Specialties.

\section{Ethics Committee Approval and Patient Consent}

This study has been approved by Sun Yat-sen University review board or ethics committee in accordance with the principles of Declaration of Helsinki.

\section{Competing Interests}

The authors have declared that no competing interest exists.

\section{References}

1. Parkin DM, Bray F, Ferlay J, et al. Global cancer statistics, 2002. CA Cancer J Clin. 2005; 55:74-108.

2. Roder DM. The epidemiology of gastric cancer. Gastric Cancer. 2002; 5:5-11.

3. Yoo CB, Jones PA. Epigenetic therapy of cancer: past, present and future. Nat Rev Drug Discov. 2006; 5:37-50.

4. Zheng L, Wang L, Ajani J, et al. Molecular basis of gastric cancer development and progression. Gastric Cancer. 2004; 7:61-77.

5. Maehama T, Dixon JE. The tumor suppressor, PTEN/MMAC1, dephosphorylates the lipid second messenger, phosphatidylinositol 3,4,5-trisphosphate. J Biol Chem. 1998; 273:13375-8.

6. Stambolic V, Suzuki A, de la Pompa JL, et al. Negative regulation of PKB/Akt-dependent cell survival by the tumor suppressor PTEN. Cell. 1998; 95:29-39.

7. Mukhopadhyay UK, Mooney P, Jia L, et al. Doubles game: Src-Stat3 versus p53-PTEN in cellular migration and invasion. Mol Cell Biol. 2010; 30:4980-95.

8. Nogueira C, Kim KH, Sung $\mathrm{H}$, et al. Cooperative interactions of PTEN deficiency and RAS activation in melanoma metastasis. Oncogene. 2010; 29:6222-32.

9. Waite KA, Eng C. Protean PTEN: form and function. Am J Hum Genet. 2002; 70:829-44.

10. Lee H, Choi SK, Ro JY. Overexpression of DJ-1 and HSP90a, and loss of PTEN associated with invasive urothelial carcinoma of urinary bladder: Possible prognostic markers. Oncol Lett. 2012; 3:507-12.

11. Kang YH, Lee HS, Kim WH. Promoter methylation and silencing of PTEN in gastric carcinoma. Lab Invest. 2002; 82:285-91.

12. Zhu X, Qin X, Fei M, et al. Loss and reduced expression of PTEN correlate with advanced-stage gastric carcinoma. Exp Ther Med. 2013; 5:57-64.

13. Liu H, Wang M, Li M, et al. Expression and role of DJ-1 in leukemia. Biochem Biophys Res Commun. 2008; 375:477-83

14. Arnouk H, Merkley MA, Podolsky RH, et al. Characterization of molecular markers indicative of cervical cancer progression. Proteomics Clin Appl. 2009; 3:516-27.

15. Liu S, Yang Z, Wei H, et al. Increased DJ-1 and its prognostic significance in hepatocellular carcinoma. Hepatogastroenterology. 2010; 57:1247-56.

16. Zhu XL, Wang ZF, Lei WB, et al. DJ-1: a novel independent prognostic marker for survival in glottic squamous cell carcinoma. Cancer Sci. 2010; 101:1320-5.

17. Kim RH, Peters M, Jang Y, et al. DJ-1, a novel regulator of the tumor suppressor PTEN. Cancer Cell. 2005; 7:263-73.

18. Tian M, Cui YZ, Song GH, et al. Proteomic analysis identifies MMP-9, DJ-1 and $A 1 B G$ as overexpressed proteins in pancreatic juice from pancreatic ductal adenocarcinoma patients. BMC Cancer. 2008,8:241.

19. Le Naour F, Misek DE, Krause MC, et al. Proteomics-based identification of RS/DJ-1 as a novel circulating tumor antigen in breast cancer. Clin Cancer Res. 2001; 7:3328-35.

20. Shimwell NJ, Ward DG, Mohri Y, et al. Macrophage migration inhibitory factor and DJ-1 in gastric cancer: differences between high-incidence and low-incidence areas. Br J Cancer. 2012; 107:1595-601.

21. Lauwers GY, Carneiro F, Graham DY, et al. Gastric carcinoma. In: Bosman FT, Carneiro F, Hruban RH, et al, eds. WHO classification of tumors of the digestive system. Lyon: IARC; 2010: 48-58.

22. Edge SB, Byrd DR, Compton CC, et al. AJCC cancer staging manual (7th edition). New York, USA: Springer; 2010.

23. Kallioniemi OP, Wagner U, Kononen J, et al. Tissue microarray technology for high-throughput molecular profiling of cancer. Hum Mol Genet. 2001; 10:657-62.

24. MacKeigan JP, Clements CM, Lich JD, et al. Proteomic profiling drug-induced apoptosis in non-small cell lung carcinoma: identification of RS/DJ-1 and RhoGDIalpha. Cancer Res. 2003; 63:6928-34.

25. Oda M, Makita M, Iwaya K, et al. High levels of DJ-1 protein in nipple fluid of patients with breast cancer. Cancer Sci. 2012; 103:1172-6.

26. He XY, Liu BY, Yao WY, et al. Serum DJ-1 as a diagnostic marker and prognostic factor for pancreatic cancer. J Dig Dis. 2011; 12:131-7.

27. Yuen HF, Chan YP, Law S, et al. DJ-1 could predict worse prognosis in esophageal squamous cell carcinoma. Cancer Epidemiol Biomarkers Prev. 2008; 17:3593-602.

28. Fan J, Ren H, Jia N, et al. DJ-1 decreases Bax expression through repressing p53 transcriptional activity. J Biol Chem. 2008; 283:4022-30.

29. Clements CM, McNally RS, Conti BJ, et al. DJ-1, a cancer- and Parkinson's disease-associated protein, stabilizes the antioxidant transcriptional master regulator Nrf2. Proc Natl Acad Sci USA. 2006; 103:15091-6.

30. Geiger TR, Peeper DS. Metastasis mechanisms. Biochim Biophys Acta. 2009; 1796:293-308.

31. Pardo M, García A, Thomas B, et al. The characterization of the invasion phenotype of uveal melanoma tumour cells shows the presence of MUC18 and HMG-1 metastasis markers and leads to the identification of DJ-1 as a potential serum biomarker. Int J Cancer. 2006; 119:1014-22.

32. He X, Zheng Z, Li J, et al. DJ-1 promotes invasion and metastasis of pancreatic cancer cells by activating SRC/ERK/uPA. Carcinogenesis. 2012; 33:555-62.

33. Bignell GR, Greenman CD, Davies H, et al. Signatures of mutation and selection in the cancer genome. Nature. 2010; 463:893-8.

34. Carracedo A, Alimonti A, Pandolfi PP. PTEN level in tumor suppression: how much is too little? Cancer Res. 2011; 71:629-33. 
35. Alessi DR, James SR, Downes CP, et al. Characterization of a 3-phosphoinositide-dependent protein kinase which phosphorylates and activates protein kinase Balpha. Curr Biol. 1997; 7:261-9.

36. Song G, Ouyang G, Bao S. The activation of Akt/PKB signaling pathway and cell survival. J Cell Mol Med. 2005; 9:59-71.

37. Yang Y, Gehrke S, Haque ME, et al. Inactivation of Drosophila DJ-1 leads to impairments of oxidative stress response and phosphatidylinositol 3-kinase/Akt signaling. Proc Natl Acad Sci USA. 2005; 102:13670-5.

38. Aleyasin H, Rousseaux MW, Marcogliese PC, et al. DJ-1 protects the nigrostriatal axis from the neurotoxin MPTP by modulation of the AKT pathway. Proc Natl Acad Sci USA. 2010; 107:3186-91.

39. Kim BS, Cho SW, Min SK, et al. Differences in prognostic factors between early and advanced gastric cancer. Hepatogastroenterology. 2011; 58:1032-40. 